\title{
Review
}

Ananda Das, Kyuyoung Bae and Wounjhang Park*

\section{Enhancement of upconversion luminescence using photonic nanostructures}

https://doi.org/10.1515/nanoph-2020-0159

Received February 29, 2020; accepted March 27, 2020

Abstract: Lanthanide-based upconversion materials convert low energy infrared photons into high energy visible photons. These materials are of interest in a myriad of applications such as solar energy harvesting, color displays and photocatalysis. Upconversion nanoparticles (UCNPs) are also of interest in biological applications as bioimaging and therapeutic agents. However, the intrinsic conversion efficiency of UCNPs remains low for most applications. In this review, we survey the recent work done in increasing the upconversion emission by changing the local electric field experienced by the UCNPs using photonic nanostructures. We review both the underlying theory behind this photonic manipulation as well as experimental demonstrations of enhancement. We discuss the recent developments in the more common plasmonic designs as well as the emerging field of dielectric nanostructures. We find that improvements in design and fabrication of these nanostructures in the last few years have led to reported enhancements of over three orders of magnitude. This large enhancement has been achieved in not only nanostructures on films but also in nanostructures that can be dispersed into solution which is especially relevant for biological applications.

Keywords: energy transfer; lanthanide ion; luminescence upconversion; nanoparticle; photonic crystal; surface plasmon.

*Corresponding author: Wounjhang Park, Department Electrical, Computer \& Energy Engineering, University of Colorado, Boulder, CO, 80309, USA; and Materials Science \& Engineering Program, University of Colorado, Boulder, CO, 80309, USA,

E-mail:won.park@colorado.edu. https://orcid.org/0000-00020806-7168

Ananda Das: Department of Physics, University of Colorado, Boulder CO, 80309, USA

Kyuyoung Bae: Department Electrical, Computer \& Energy Engineering, University of Colorado, Boulder, C0, 80309, USA

Ә Open Access. ( 2020 Ananda Das et al., published by De Gruyter (cc) BY License.

\section{Introduction}

Upconversion luminescence (UCL) is a fluorescence mechanism that converts two or more lower energy photons into a higher energy photon $[1,2]$. This process occurs through the interaction between the low energy incident photons and long-lived intermediate state of the luminescent material. Through processes such as energy transfer, excited state absorption and triplet-triplet annihilation, the material is excited to a higher energy level and subsequently emits a high energy photon. Compared to other frequency conversion mechanisms such as high harmonic generation, optical parametric oscillation or four-wave mixing that stem from materials with nonlinear optical susceptibilities, this type of upconversion occurs at much lower incident intensities and does not need to satisfy stringent phase matching conditions.

A typical upconversion material consists of an inert host matrix doped with rare Earth ions such as $\mathrm{Yb}^{3+}, \mathrm{Er}^{3+}$, and $\mathrm{Tm}^{3+}$. The lanthanide based upconversion materials have a rich energy level landscape with easily tunable emission characteristics which is useful in the fields of display [3], anticounterfeiting [4] and fingerprinting technology [5]. Many of the ions have adjacent energy levels with small energy gaps which follow a Boltzmann distribution and as a result can be used to realize a highly sensitive optical thermometer [6]. The rare Earth based upconversion materials are also of interest for solar cell technology as the conversion of infrared light into the visible regime allows for the potential to harvest below band gap photons that comprise a significant portion of the solar spectrum [7]. This upconversion of solar radiation can also be used to enhance photocatalytic reactions [8].

Upconversion materials are also of interest for their use in biological applications. Upconversion nanoparticles (UCNPs) are commonly used as bioimaging agents. Compared to conventional fluorescent dyes which need to be excited with high energy UV light, UCNPs are excited with near infrared (NIR) photons. UCNPs have numerous advantages over the more commonly used dyes. The UV light often excites background fluorescence from the 
surrounding tissue while the infrared light does not and as a result provides a high contrast against the background. The infrared light can penetrate much deeper into the body before getting absorbed. The UCNPs do not suffer from photobleaching or photoblinking that occurs in other fluorescent materials such as quantum dots. In additional to their use in bioimaging, UCNPs can also be used as a treatment modality through methods such as photodynamic therapy $[9,10]$ and drug delivery [11].

Despite the advantages of UCNPs, the main challenge to be overcome with UCNPs is their poor efficiency. The upconversion efficiency is typically on the order of a few percent. This low efficiency stems from the forbidden nature of the f-f transition involved in the upconversion process as well as from the potential for additional nonradiative pathways introduced by defects and cross relaxation $[1,2]$. Thus, in order to make UCNPs viable in the applications mentioned above, additional steps are necessary to increase the overall brightness of the UCNPs. Enhancing emission is especially crucial in biological applications as they require much lower incident powers [12, 13].

The ways to enhanced upconversion emission can be split into two categories. The first is through nanoparticle engineering. Here, the absorption cross-section and radiative emission rates can be controlled by changing the synthesis conditions. The most common method for this type of enhancement is to grow a shell around that nanoparticle that passivates the surface and greatly reduces the number of surface defects that act as nonradiative pathways in the upconversion process [14-23]. This is especially relevant for the smaller ( $<10 \mathrm{~nm}$ ) UCNPs as their surface to volume ratio is a lot higher $[14,16]$. The shell can further be doped with a sensitizer ion such $\mathrm{Yb}^{3+}$ or $\mathrm{Nd}^{3+}$ to increase the absorption cross-section. While adding a sensitizer to the upconversion process has been a relatively standard procedure, recent work has shown that the shell can be doped with much higher concentrations of sensitizer ions before the effects of concentration quenching kick in $[18,19]$. The absorption cross-section can also be enhanced by functionalizing the UCNPs with dyes that absorb in the IR region [24-26]. Finally, the upconversion process can be enhanced by manipulating the crystal field experienced by the ions by doping with a wide variety of ions such as $\mathrm{Bi}$ [27], Cd [28], Fe [29, 30], K [31] and Li [32-34].

The second way to enhance upconversion, and the focus of this review, is through photonic engineering. Here, the effective absorption cross-section is increased by increasing the local photon density of states. Note that the nanoparticle engineering techniques mentioned previously can be used in conjunction with these methods to achieve maximum enhancement. Using plasmonic nanostructures, it is possible to achieve over $10^{2}$ fold enhancement of the local field. Since the upconversion process is a multiphoton process, this can potentially lead to a $10^{2 n}$ increase in emission where $\mathrm{n}$ is the number of photons involved in upconversion, resulting in a significantly brighter UCNP.

In this review, we assess the recent work on the photonic engineering of the local density of states to enhance upconversion emission. We start by establishing underlying theory behind photonic engineering and show how the absorption cross-section, decay rates and energy transfer rates can all be affected by photonic nanostructures. Further analysis of the rate equations involved shows that a multiphoton process can be greatly enhanced by increasing the local $\mathrm{E}$ field experienced by the particle. We then do an indepth survey the enhancement from photonic nanostructures reported in last few years. We survey both traditional plasmonic nanostructures as well as the emerging field of dielectric photonic nanostructures. We conclude with observations on the future prospects of the field.

\section{Theory}

For the theory section of this review, we will focus specifically on Energy Transfer Upconversion (ETU) which is known to be the most efficient upconversion mechanism [1]. However, the theoretical framework presented here can easily be extended to other upconversion mechanisms such as excited state absorption or triplet-triplet annihilation.

There are three main process involved in ETU: absorption, decay (radiative and nonradiative), and energy transfer. Absorption cross-section is defined as the ratio of absorbed power to the incident intensity [35].

$$
\sigma=\frac{P_{a b s}}{I_{\text {inc }}}=c \mu_{0} \omega \operatorname{Im}\{\alpha\} \frac{\left|\hat{\boldsymbol{n}}_{d} \cdot \boldsymbol{E}\left(\boldsymbol{r}_{0}\right)\right|^{2}}{\left|E_{0}\right|^{2}}
$$

where $\alpha$ is atomic polarizability, $\boldsymbol{E}\left(\boldsymbol{r}_{0}\right)$ is the local field at the position of the quantum emitter and $\boldsymbol{E}_{0}$ is the incident field. The polarizability is determined by the transition matrix element and thus depends only on the electronic wavefunctions and not on the photonic nanostructures. The local field, however, can be strongly enhanced by a suitably designed photonic nanostructure, thereby impacting the absorption cross-section. Specifically, equation (1) shows absorption cross section scales as $\left|E / E_{0}\right|^{2}$, that is, linearly with the local intensity. 
Photonic nanostructures can affect both radiative and nonradiative decay rates. According to Femi's golden rule, the radiative transition rate is determined by the transition matrix element and the density of states. In quantum electrodynamics, we should consider the states of the combined system of atom and photon and therefore the density of states includes the photon states as well as the electronic states. Consequently, the large increase in photon density of states by a nanophotonic structure can increase the optical transition rate. This is the well-known Purcell effect that has been widely studied in photonics $[36,37]$.

Nonradiative decay such as multiphonon emission is not directly impacted by the local field. However, the presence of a lossy medium, e. g., metal, can introduce additional nonradiative decay channels, increasing the overall nonradiative decay rate and consequently resulting in luminescence quenching. Just like the radiative decay rate, non-radiative decay rates can also be estimated by the dyadic Green function [35]. The tradeoff between the positive effect of Purcell effect and the negative effect of quenching is an important aspect of photonic engineering of quantum emitter. Typically, quenching dominates at short distances while the Purcell effect remains pronounced at larger distances [38, 40], as shown in Figure 1A and B. It is therefore customary to insert a thin $(\sim 10 \mathrm{~nm})$ spacer layer between metal surface and quantum emitters.

Finally, the rate of Förster energy transfer can also be calculated by using the dyadic Green function [41, 42]. An earlier study observed enhanced Förster energy transfer rate in optical cavities and attributed it to the enhanced local density of states to which the donor emission rate is directly proportional [41]. However, it was later shown that the energy transfer enhancement is not related to the density of states enhancement [43, 44]. Theoretical studies indicate that the energy transfer enhancement is generally smaller than the spontaneous emission enhancement, although the enhancement depends strongly on the donoracceptor distance [42]. Our calculations for a donoracceptor pair in the vicinity of a silver surface showed that the energy transfer rate can be enhanced at frequencies slightly lower than the surface plasmon frequency and quenched at slightly higher frequencies (Figure 1C) [35, 39]. Also, both the enhancement factor and the bandwidth over which enhancement is achieved are smaller than the fluorescence enhancement. Enhanced energy transfer rate in a plasmonic nanostructure has recently been observed experimentally by a time-resolved photoluminescence spectroscopy study [45].

For a complete understanding of the ETU mechanism, we need to set up a system of rate equations containing all involved states and transitions. As an example, we will consider the prototypical $\mathrm{Yb}^{3+}-\mathrm{Er}^{3+}$ system whose energy level scheme is shown in Figure 1D. As the details of the rate equation analysis have been published elsewhere [35, 39], we simply present the results here. Analytical solutions can be found in two limiting cases: weak and strong excitation regimes. In the strong excitation regime, the intermediate energy levels are highly populated and upconversion occurs readily with one-photon absorption. Therefore, the overall
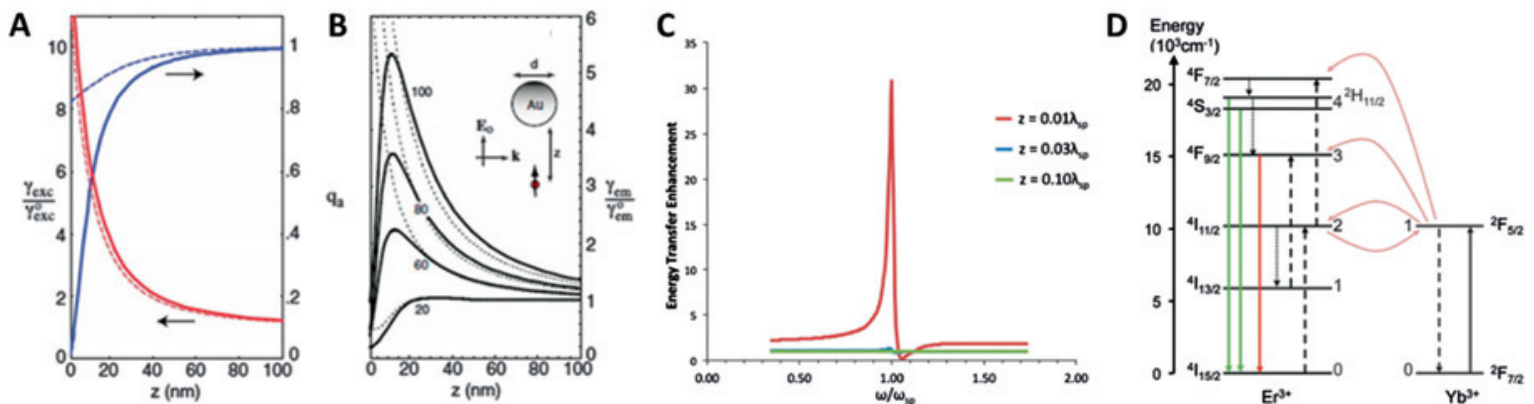

Figure 1: $(A, B)$ Calculated quantum yield $q_{a}$, excitation rate $\gamma_{\mathrm{exc}}$, and fluorescence rate $\gamma_{\mathrm{em}}$ as a function of the separation distance between gold nanoparticle and fluorescent molecule. $\gamma_{\mathrm{exc}}$ and $\gamma_{\mathrm{em}}$ are normalized with their corresponding free-space values $(z \rightarrow \infty)$. The solid curves are the result of multiple multipole calculations [38] whereas the dashed curves correspond to the dipole approximation in which all higher order multipoles of gold nanoparticles were ignored. In (A) the particle diameter is $\mathrm{d}=80 \mathrm{~nm}$ and in (B) it is indicated in the figure. Excitation wavelength is $\lambda=650 \mathrm{~nm}$ and $\varepsilon=-12.99+i 1.09$ (gold) (C) Energy transfer rate enhancement factor calculated for a donor-acceptor pair with a pair separation of $3.4 \mathrm{~nm}$ placed at various distances, $z$, from a flat silver surface. $\omega_{\mathrm{sp}}$ and $\lambda_{\text {sp }}$ represent the surface plasmon frequency and wavelength, respectively (D) Energy levels of $\mathrm{Yb}^{3+}$ and $\mathrm{Er}^{3+}$ ions relevant to the ETU process. Initial absorption is indicated by the black solid line arrow, subsequent energy transfer processes by the dashed arrows, non-radiative relaxations by dotted arrows and the final upconverted luminescence by the green and red arrows. Adapted from Ref. [38] for (A, B) with permission from Anger et al., Phys. Rev. Lett. vol. 96, p. 113002, 2006, ( 2006 by the American Physical Society, and from Ref. [39] for (C, D) with permission from Lu et al., ACS Nano vol. 8, pp. 77807792, 2014, (C) 2014 by the American Chemical Society. 
enhancement factor for upconversion is simply equal to the absorption enhancement factor,

$$
F_{\text {strong }} \approx F_{a}
$$

In the weak excitation regime where the intermediate energy levels are nearly empty, upconversion requires two back-to-back photon absorption and energy transfer within the lifetime of the intermediate energy level. Thus, the enhancement factor contains all three components: absorption, energy transfer and decay from the intermediate energy level

$$
F_{\text {weak }} \approx \frac{F_{d 4} F_{a}^{2}}{F_{D 10}^{2}}
$$

Here $\mathrm{F}_{\mathrm{d} 4}$ is the enhancement factor for the rate of second energy transfer process that excites the $\mathrm{Er}^{3+}$ ion into the emitting level, $\mathrm{F}_{\mathrm{D} 10}$ is the enhancement factor for the decay rate of $\mathrm{Yb}^{3+}$ ion and $\mathrm{F}_{\mathrm{a}}$ is the absorption enhancement factor. The requirement of two back-to-back absorption manifests itself as the quadratic dependence on $F_{a}$. The decay of intermediate energy level, whether radiatively or non-radiatively, detrimentally impacts upconversion and thus $\mathrm{F}_{\mathrm{D} 10}$ shows up in the denominator.

Based on the analysis given above, we may draw a few conclusions. First, even with extremely high Purcell enhancement, the green emission would not be enhanced significantly. This is because the ETU is usually limited by the excitation process and not by the radiative decay. Indeed, equations (2) (3) show that the processes occurring at the excitation wavelength, which are absorption, energy transfer and the donor decay, are important for the overall enhancement of upconversion. It is therefore desirable to tune the photonic resonance to the excitation wavelength rather than the emission wavelength. Furthermore, in the weak excitation limit, the upconverted luminescence intensity is enhanced linearly to energy transfer enhancement and quadratically to absorption enhancement. Thus, absorption enhancement is expected to produce the most dramatic improvement in upconversion. Finally, we note that this analysis can be extended to 3- or 4-photon upconversion processes observed in, e. g., $\mathrm{Yb}^{3+}-\mathrm{Tm}^{3+}$ system. It is generally expected the overall enhancement factor should scale as $F_{a}^{n}$ in the weak excitation regime and as $F_{a}$ in the strong excitation regime, where $n$ is the number of photons involved in upconversion. Thus, the absorption enhancement will make even more dramatic enhancement in the overall upconversion in these higher order upconversion system.

\section{Experimental demonstrations of upconversion enhancement}

Figure 2 shows the reported enhancement of the upconverted green emission from plasmonic nanostructures since 2015. For publications prior to this point, we refer to our previous review paper where we performed an exhaustive study of reported plasmonic enhancement at the time [35]. The enhancement factor is plotted against the peak of the surface plasmon resonance (SPR). Note that though several structures report multi-peak or broadband resonances that cover both absorption and emission wavelengths [46-49], for simplicity we only focus on the primary peak of the resonance. The results are also summarized in full detail in Table 1 below.

A few things of note are immediately evident. First, a majority of the plasmonic nanostructures target the infrared wavelengths over visible wavelengths. This is a significant shift from our study in 2015 where we found most nanostructures had resonances at visible wavelengths [35]. The shift towards infrared wavelengths is due

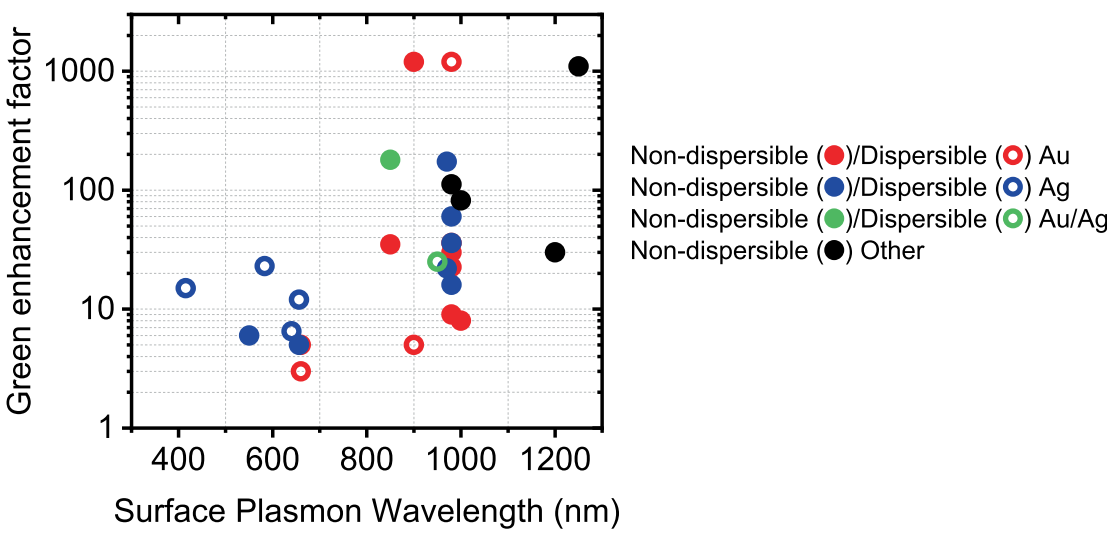

Figure 2: Survey of plasmonic enhancement of upconversion from 2015 onwards. The data is divided by material choice for the nanostructure (red for $\mathrm{Au}$, blue for $\mathrm{Ag}$, green for Au-Ag alloy, and black for other materials) as well as by whether the nanostructure can be dispersed into solution or not (filled circle for nondispersible nanostructures and unfilled circle for dispersible). All references are listed in Table 1. 
Table 1: Upconversion enhancement factors for various method, materials, target SPR wavelength, emission lines and dispersibility for plasmon enhancement. RE, D, ND, MIM, NP, NW, NR, and OPC represent rare Earth, dispersible, non-dispersible, metal-insulator-metal, nanoparticle, nanowire, nanorod, and opal photonic crystal respectively.

\begin{tabular}{|c|c|c|c|c|c|c|c|}
\hline Method & SP Material & RE & SPR (nm) & Green & Red & Dispersibility & Ref. \\
\hline \multicolumn{8}{|l|}{ Plasmonic } \\
\hline Au-Ag nanocage & Gold/silver & $\mathrm{Er}$ & 950 & 25 & & $\mathrm{D}$ & {$[65]$} \\
\hline Au shell@UCNP/SiO ${ }_{2}$ & Gold & $\mathrm{Er}$ & 900 & 5 & & $\mathrm{D}$ & [70] \\
\hline AuNR@SiO ${ }_{2} @ U C N P$ & Gold & Er & 660 & 3 & 6.7 & $\mathrm{D}$ & {$[71]$} \\
\hline Ag@UCNP & Silver & $\mathrm{Er}$ & 656 & 12 & 9 & $\mathrm{D}$ & [68] \\
\hline Ag NP decorated UCNP@SiO & Silver & $\mathrm{Er}$ & 640 & 6.5 & 5.5 & $\mathrm{D}$ & [69] \\
\hline Au nanocap & Silver & $\mathrm{Er}$ & 583 & 23 & 43 & $\mathrm{D}$ & {$[64]$} \\
\hline $\begin{array}{l}\text { UCNP@SiO }{ }_{2} @ A g \\
\text { MIM }\end{array}$ & Silver & Ho & 415 & 15 & 7.5 & $\mathrm{D}$ & {$[67]$} \\
\hline Au disk/UCNP/Au disk & Gold & $\mathrm{Er}$ & 980 & 1200 & & $\mathrm{D}$ & {$[66]$} \\
\hline $\begin{array}{l}\text { Ag disk/UCNP/Ag film } \\
\text { NPs with film - patterned }\end{array}$ & Silver & $\mathrm{Er}$ & $970,659,549$ & 174 & & ND & {$[47]$} \\
\hline $\mathrm{Cu}_{2-x} \mathrm{~S}$ NPs film & $\mathrm{Cu}_{2-x} \mathrm{~S}$ & $\mathrm{Er}$ & 1000 & 200 & & ND & {$[52]$} \\
\hline Au NRs on substrate & Gold & Er & 980 & 22.6 & & ND & {$[57]$} \\
\hline Au nanocavity & Gold & $\mathrm{Er}$ & 980 & 30 & & ND & [59] \\
\hline Au NRs layer & Gold & $\mathrm{Er}$ & 850 & 35 & & ND & {$[56]$} \\
\hline Au NRs on substrate & Gold & $\mathrm{Er}$ & 660 & 5 & 10.6 & ND & {$[55]$} \\
\hline Au sandwich & Gold & $\mathrm{Er}$ & 1000 & 8 & & ND & [58] \\
\hline $\begin{array}{l}\text { Ag grating } \\
\quad \text { - unpatterned }\end{array}$ & Silver & Er & 980 & 16 & 39 & ND & [45] \\
\hline $\mathrm{W}_{18} \mathrm{O}_{49} \mathrm{NWs}$ on FTO film & $\mathrm{W}_{18} \mathrm{O}_{49}$ & $\mathrm{Er}$ & $980,521,545,660$ & 112 & & ND & {$[48]$} \\
\hline $\mathrm{Cu}_{2-x} \mathrm{~S} @ \mathrm{SiO}_{2}$ & $\mathrm{Cu}_{2-x} \mathrm{~S}$ & $\mathrm{Er}$ & 1250 & 1100 & & ND & {$[50]$} \\
\hline $\mathrm{mCu}_{2-\mathrm{x}} \mathrm{S} @ \mathrm{SiO}_{2} @ U C N P$ in $\mathrm{TiO}_{2}$ film & $\mathrm{mCu}_{2-\mathrm{x}} \mathrm{S}$ & $\mathrm{Er}$ & 1200 & 30 & & ND & {$[51]$} \\
\hline Au film on microfiber & Gold & Er & 980 & 36 & & ND & {$[53]$} \\
\hline Ag nanoplatelets on substrate & Silver & Er & $\begin{array}{l}970 \\
656\end{array}$ & $\begin{array}{l}22 \\
5\end{array}$ & & ND & {$[60]$} \\
\hline Au NRs/UCP@SiO film & Gold & $\mathrm{Er}$ & 980 & 9 & & ND & [61] \\
\hline porous Ag film & Silver & $\mathrm{Er}$ & $400-980$ & 36 & & ND & [49] \\
\hline Au-Ag alloy film & Gold/silver & $\mathrm{Er}, \mathrm{Tm}$ & 850 & 180 & & ND & {$[46]$} \\
\hline $\begin{array}{l}\text { porous Ag film } \\
\text { OPC }\end{array}$ & Silver & $\mathrm{Er}$ & 980 & 60 & 50 & ND & [62] \\
\hline Au NR/OPC & Gold & $\mathrm{Er}$ & 900 & 1200 & 3000 & ND & [54] \\
\hline Inverse OPC & Silver & $\mathrm{Er}$ & 550 & 6 & 4 & ND & [63] \\
\hline Dielectric & & & & & & & \\
\hline Four-silicon-pillar metasurface & $\mathrm{Si}$ & $\mathrm{Tm}$ & 965,800 & 400 & & ND & {$[72]$} \\
\hline $\mathrm{Si}_{3} \mathrm{~N}_{4}$ photonic crystal & $\mathrm{Si}_{3} \mathrm{~N}_{4}$ & $\mathrm{Er}$ & 980 & 130 & 350 & ND & [73] \\
\hline Polymeric microbead & PEGDA & $\mathrm{Er}, \mathrm{Tm}, \mathrm{Eu}$ & & $\begin{array}{c}\text { Eu-103000 } \\
\text { Er-100 }\end{array}$ & & ND & [74] \\
\hline
\end{tabular}

to the superior quadratic scaling that can be achieved by enhancing the effective absorption coefficient as discussed earlier in section 2. Indeed, the nanostructures with resonances in the infrared display much higher enhancements on average than those with resonances in the visible regime.

The structures are predominantly made from the noble metals, gold and silver. These materials can be easily processed into nanostructures and, in the case of the dispersible structures, have a localized surface plasmon resonance (LSPR) at visible wavelengths. Other plasmonic semiconductor materials such as $\mathrm{Cu}_{2-\mathrm{x}} \mathrm{S}$ NPs and $\mathrm{W}_{18} \mathrm{O}_{49}$ nanowires have also emerged as viable options due to their unique properties [48, 50-52]. A large number of highly mobile hole carriers in $\mathrm{Cu}_{2-\mathrm{x}} \mathrm{S}$ NPs support a strong LSPR in the near-infrared and broadband LSPR across the visible and NIR regions of $\mathrm{W}_{18} \mathrm{O}_{49}$ nanowires (NWs) enhances both excitation and emission of UCNPs. The use of dielectric materials to enhance upconversion emission has also emerged as a viable alternative to plasmonic designs. In 
contrast to the plasmonic materials, these nanostructures do not introduce new nonradiative pathways to the upconversion process and thus are not subject to plasmonic quenching.

Figure 2 further splits the data into nanostructures that can be dispersed into solution versus those that are fixed onto a film. As discussed earlier, the applications of these two types of structures are very different from each other. The applications of the non-dispersible plasmonic structures include multicolor flexible display, fingerprint, solar energy harvesting, temperature detection, and photocatalysis. Dispersible plasmonic structures on the other hand are of interest for biological applications where they can be used for bioimaging and therapeutic techniques such as photodynamic therapy and drug delivery. In the next sections, we take a deeper look at the two types of nanostructures and discuss the publications in each case that were able to achieve significant enhancement of upconversion emission. We end with a discussion of reported enhancement from the few dielectric nanostructures that have recently emerged as an alternative to the traditional plasmonic designs.

\subsection{Non dispersible plasmonic enhancement}

Figure 3 shows recently reported non-dispersible plasmonic nanostructures, presented as squares in Figure 2. Many methods are applied to harness the efficient plasmonic enhancement. While the more traditional approach is to use UCNPs on patterned [45, 47, 55-59] and unpatterned [48, 50-53, 60, 61] plasmonic films, new techniques involving porous metal films $[46,49,62]$ and opal photonic crystals $[54,63]$ (OPC) have emerged in recent years. Porous metal films were used as they are able to support broad SPRs that cover both the excitation and emission wavelengths. Over 1000-fold enhancement was achieved by OPCs embedded with metal nanostructures [54]. Here, the luminescent intensity of UCNPs can be exceedingly improved by combining local field enhancement from both the OPC and the SPRs of the metal nanoparticles.

Zhang et al. demonstrated a 36x improvement in the UCL from $\beta-\mathrm{NaYF}_{4}: \mathrm{Yb}^{3+}, \mathrm{Er}^{3+}$ UCNPs using a microfiber coated with an Au nanofilm as shown in Figure 3A [53]. By controlling the pump power and the UCNP concentration, they were able to achieve a wide-range temperature detection of $486 \mathrm{~K}(325-811 \mathrm{~K})$ with a temperature resolution of $0.035-0.046 \mathrm{~K}$. Using the SPR of the Au nanofilm on a microfiber, they were able to improve the fibercoupled optical temperature sensing properties of the UCNPs.

Lee et al. designed a metal-insulator-metal (MIM) structure (Figure 3B) using Ag nanodisks on a Ag film to enhance the UC luminescence of an intermediate UCNP film [47]. The structure was also designed to enhance the downshifting (DS) luminescence of a $\mathrm{Tb}(\mathrm{thd})_{3}$ film that coated the top of the structure. Using the strong field enhancement from the gap plasmon mode of the structure, they were able to achieve a 174x and 29x enhancement of UC and DS luminescence, respectively. This approach offers the possibility of using the full solar spectrum in

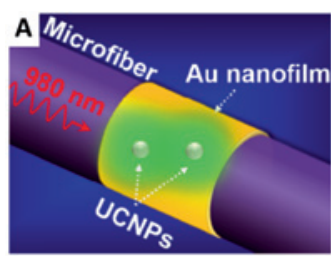

B

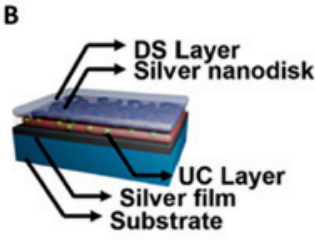

C

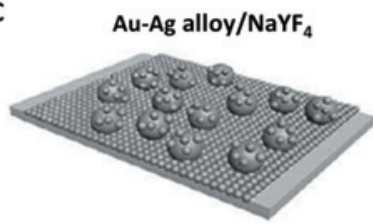

D

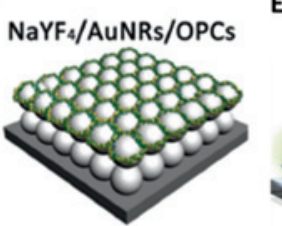

E

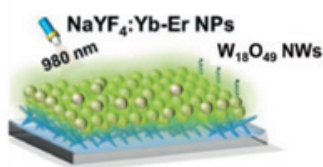

$\mathbf{F}$

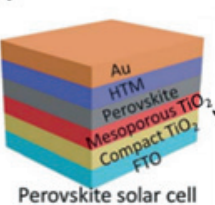

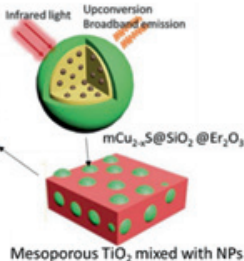

Figure 3: Non-dispersible plasmonically enhanced nanostructures (A) Schematic of the UCNPs/Au structure. A microfiber is coated by a sputtered Au nanofilm and drop-casted $\mathrm{NaYF}_{4}: \mathrm{Yb}^{3+}, \mathrm{Er}^{3+}$ UCNPs (B) Schematic of an MIM structure with UC and DS layers. The silver nanodisks are fabricated by a nanoimprinting method (C) Schematic illustrations of porous $\mathrm{Au}-\mathrm{Ag}$ / $\mathrm{NaYF}_{4}: \mathrm{Yb}^{3+}, \mathrm{Tm}^{3+}$ composite film (D) Schematic illustration of $\mathrm{NaYF}_{4}, \mathrm{Er} / \mathrm{AuNRs}$ / OPCs surface plasmonic photonic crystal hybrid structure (E) Schematics of a $\mathrm{NaYF}_{4}: \mathrm{Yb}^{3+}, \mathrm{Er}^{3+} / \mathrm{W}_{18} \mathrm{O}_{49}$ film on FTO glass (F) Schematic illustration on the

$\mathrm{mCu}_{2-\mathrm{x}} \mathrm{S} @ \mathrm{SiO}_{2} @ \mathrm{Er}_{2} \mathrm{O}_{3}$ nanocomposite and their application in perovskite solar cell. Reproduced from Ref. [53] for (A) with permission from Zhang et al., ACS Appl. Mater. Interfaces vol. 9, pp. 42935-42942, 2017, (C 2017 by the American Chemical Society, from Ref. [47] for (B) with permission from Lee et al., Nano Lett. vol. 15, pp. 2491-2497, 2015, (C) 2015 by the American Chemical Society, from Ref. [46] for (C) with permission from Chen et al., Adv. Funct. Mater. vol. 25, pp. 5462-5471, 2015, ( 2015 WILEY-VCH Verlag GmbH \& Co. KGaA, Weinheim, from Ref. [54] for (D) with permission from Yin et al., Adv. Mater. vol. 28, pp. 2518-2525, 2016, (C) 2016 WILEY-VCH Verlag GmbH \& Co. KGaA, Weinheim, from Ref. [48] for (E) with permission from Zhang et al., Adv. Sci. vol. 5, pp. 1800748, 2018, (C) 2018 The Authors. Published by WILEYVCH Verlag GmbH \& Co. KGaA, Weinheim, and from Ref. [50] for (F) with permission from The Royal Society of Chemistry. 
photovoltaic devices to maximize the power conversion efficiency.

Alloys of noble metals can also be used to achieve efficient UCL. Chen et al. used a porous film of $\mathrm{Au}-\mathrm{Ag}$ alloy islands to enhance the UCL of $\mathrm{NaYF}_{4}: \mathrm{Yb}^{3+}, \mathrm{Tm}^{3+} \mathrm{UCNPs}$ (Figure 3C) [46]. The $\mathrm{Au}-\mathrm{Ag}$ alloy structure is advantageous as its extinction cross section can be easily tuned by changing the ratio between $\mathrm{Au}$ and $\mathrm{Ag}$ and it has a higher chemical stability than pure Ag. The Au-Ag alloy was optimized by controlling the concentration of $\mathrm{AgNO}_{3}$ to suppress absorption and increase scattering in the visible spectrum as well as to absorb more NIR light. This resulted in an improved scattering to absorption ratio and consequently a UCL enhancement factor of 180x. As discussed in section 2, the 3-photon upconversion in a $\mathrm{Yb}^{3+}-\mathrm{Tm}^{3+}$ system should exhibit a cubic dependence on the excitation power while the 2-photon upconversion process of the $\mathrm{Yb}^{3+}-\mathrm{Er}^{3+}$ system scales quadratically. It is thus expected that the overall enhancement factor is much higher in $\mathrm{a} \mathrm{Yb}^{3+}-\mathrm{Tm}^{3+}$ system even with the same local field enhancement. By using the improved UCL, the authors demonstrated highresolution fingerprint identification, exhibiting excellent optical contrast and high detection sensitivity.

Figure 3D shows the nanophotonic structure fabricated by Yin et al. using gold nanorods (AuNRs) implanted three-dimensional PMMA opal photonic crystals (OPCs). In this structure, the local electric field was amplified due to the combination of photonic crystal (PC) and SPR effects [54]. The UCL from a film of UCNPs assembled on the surface of the AuNR/OPC structure was enhanced by 1200x. FDTD simulations showed a significant field enhancement (770x) at the hot spots at $980 \mathrm{~nm}$, which indicates absorption enhancement made a strong contribution to the overall UCL enhancement. Using this structure, a multicolor UCL display was demonstrated by nanoprinting onto a flexible substrate for infrared anti-counterfeiting.

Non-metal plasmonic materials have also been used to enhance UCL. As shown in Figure 3E, Zhang et al. observed an enhancement of UCL from a heterostructure film of tungsten oxide NWs [48]. The heterostructure film was fabricated via self-assembly of $\mathrm{NaYF}_{4}: \mathrm{Yb}^{3+}, \mathrm{Er}^{3+} \mathrm{NPs}$ onto the nonmetallic plasmonic layer of $\mathrm{W}_{18} \mathrm{O}_{49}$ NWs grown on a fluorine doped tin oxide (FTO) glass substrate. The broad LSPR absorption band of $\mathrm{W}_{18} \mathrm{O}_{49} \mathrm{NWs}$ arising from the oxygen vacancies on their surfaces overlapped with both the excitation and emission bands of $\mathrm{NaYF}_{4}: \mathrm{Yb}^{3+}, \mathrm{Er}^{3+} \mathrm{NPs}$. The structure exhibited a $112 \mathrm{x}$ enhancement of green emission, allowing a catalytic activity for $\mathrm{H}_{2}$ evolution from $\mathrm{BH}_{3} \mathrm{NH}_{3}$ over the heterostructure of $\mathrm{NaYF}_{4}: \mathrm{Yb}^{3+}, \mathrm{Er}^{3+}$

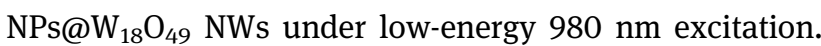
The amount of $\mathrm{H}_{2}$ evolution of the heterostructure was $35 \mathrm{x}$ higher than that of standard $\mathrm{BH}_{3} \mathrm{NH}_{3}$ hydrolysis after irradiation with $980 \mathrm{~nm}$ for $1 \mathrm{~h}$.

Significant upconversion enhancement has also been demonstrated by Zhou et al. using $\mathrm{Cu}_{2-\mathrm{x}} \mathrm{S}$ NPs (Figure 3F) [50]. These heavily doped semiconductors have a LSPR absorption band at NIR wavelengths $(800-1600 \mathrm{~nm})$. As the excitation power was increased, the authors observed a shift in the upconversion emission from the standard narrow line, multi-peak emission to a bright white broadband emission centered at $650 \mathrm{~nm}$ which they attribute to a photon or thermal avalanche [1]. This broadband emission showed an extremely high nonlinear power dependence (slope of 10), resulting in an upconversion enhancement of $1100 x$. These nanocomposites were used to achieve a 10\% increase in power conversion efficiency in perovskite solar cells. It is noted that the high nonlinearity (10th order) leads to a very large enhancement factor even with modest local field enhancement.

\subsection{Dispersible plasmonic enhancement}

Given the relevance of UCNPs in biomedical applications, it is critical that the plasmonic configuration used to enhance upconversion be both small in size and dispersible into water. Moreover, the irradiation damage threshold of the infrared light near $980 \mathrm{~nm}$ is very low, less than $726 \mathrm{~mW} \mathrm{~cm}^{-2}$ for in vivo application [12, 13]. Given the low absorption cross-section of the UCNPs, it is important to enhance the luminescence intensity/efficiency of UCNPs at low excitation intensities.

Almost all the reported dispersible enhancement mechanisms target visible wavelengths and rely on coreshell structures $[64,65,67-71]$. These structures use the LSPR of the plasmonic material to enhance the local electric field. The resonance is dependent on material properties and shape, not on arrangement or periodicity, making it ideal for biological applications. The core-shell structures can be made using gold or silver which are both commonly available, non-toxic and have LSPRs at visible wavelengths. Silver is the predominant metal of choice due to its lower damping constant compared to gold.

Enhancement factors reported for dispersible structures with resonances in the visible wavelength are typically low, on the order of 10x or lower. The reason for this is twofold. As mentioned previously, these structures use the Purcell effect to enhance emission which is not as effective as enhancing the field at the absorption wavelength. Additionally, it is hard to achieve a uniform coating of metal nanoparticles around the core upconversion particle 

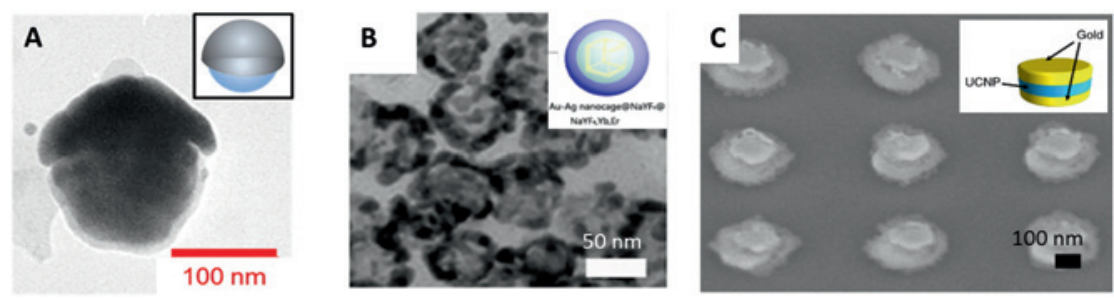

Figure 4: Water dispersible plasmonically enhanced nanostructures. a) UCNP covered with Ag nanocap. A thin layer of Ag was evaporated onto UCNPs on a quartz substrate. The UCNPs were removed via polymer stamping and dispersed into water. b) Ag-Au nanocage with intermediate UCNP layer. A UCNP layer was grown over an Ag nanocube and the whole structure was then covered with a layer of Au. c) Metal-insulator-metal nanostructure. The MIMs were fabricated lithographically by multilayer deposition on a resist hole array. Reproduced from Ref. [64] for (A) with permission from Yamamoto et al., J. Phys. Chem. C vol. 119, pp. 1175-1179, 2015, () 2015 by the American Chemical Society, from Ref. [65] for (B) with permission from Chen et al., Sci. Rep. vol. 7, p. 41079, 2017, licensed under Creative Commons Attribution 4.0 International License, and from Ref. [66] for (C) with permission from Das et al., Nat. Commun. vol. 9 , p. 4828, 2018, licensed under Creative Commons Attribution 4.0 International License.

which affects the overall measured enhancement of the particles on average.

Of the structures targeting emission wavelengths, Yamamoto et al. were able to achieve the highest enhancement, enhancing the UCNP green emission by a factor of $23 \mathrm{x}$ and the red emission by a factor of $43 \mathrm{x}$ using UCNPs with Ag nanocaps (Figure 4A) [64]. The structures were made by dispersing UCNPs onto a quartz substrate and evaporating Ag, covering the top half of all the particles. The particles were then removed from the substrate via polymer stamping and dispersed into solution. Compared to other metal seed growth-based coating methods $[67,68,71]$, this process resulted in a more uniform coating and thus likely a higher enhancement.

There are much fewer water-dispersible plasmonic structures that target the infrared wavelengths. This is because it is difficult to engineer resonances at these wavelengths while also restricting the size of the nanostructures and requiring that they be dispersed into solution. The nanostructures mentioned in the section 3.1 typically have feature sizes on the order of the resonance wavelength which is too large for biological applications and also sometimes rely on the periodicity of the overall structure [45, 47, 54, 58, 59, 63]. However, with careful design consideration, it is still possible to make waterdispersible plasmonic nanostructures with resonances at the infrared wavelengths.

Chen et al. designed a dispersible Ag-Au nanocage structure with a resonance in the NIR region (Figure 4B) [65]. The structure consists of an $\mathrm{Ag}$ nanocube with an intermediate spacer layer of $\mathrm{NaYF}_{4}$ grown around it to reduce plasmonic quenching. Another layer of luminescent $\mathrm{NaYF}_{4}: \mathrm{Yb}^{3+}, \mathrm{Er}^{3+}$ is grown around the inert $\mathrm{NaYF}_{4}$ and the whole structure is then covered with a layer of $\mathrm{Au}$ via a galvanic replacement reaction between the $\mathrm{Ag}$ nanocubes and $\mathrm{HAuCl}_{4}$ solution. Without the Au outer coating, the nanostructure has a SPR at $430 \mathrm{~nm}$ and shows an enhancement of $7 x$. With the additional Au coating, the resonance redshifts to $950 \mathrm{~nm}$ and the maximum enhancement of $25 \mathrm{x}$ is achieved.

Another dispersible nanostructure targeting the NIR region was a metal-insulator-metal (MIM) nanostructure designed and fabricated by our group (Figure 4C) [66]. The structure is comprised of a UCNP disk sandwiched between two $\mathrm{Au}$ disks and uses a gap plasmon resonance to greatly enhance the electric field within the UCNP layer of the structure. The structures were lithographically fabricated and thus highly uniform. The MIM nanostructure is unique in that its resonance relies on geometric parameters, specifically the diameter and thickness of the I layer, and thus the resonance can be easily tuned to any desired wavelength simply by changing the size of the structure. The plasmon resonance supported by the MIM nanostructure does not depend on the period of the structure so, even when dispersed into solution, a strong localized electric field can be achieved. Using an MIM nanostructure tuned to $980 \mathrm{~nm}$, we measured a 1200x enhancement from the MIMs while still attached to the Si substrate. The MIMs were then dispersed into water, incubated into cancer cells and imaged using a confocal microscope set up. Due to their enhanced upconversion, we observed similar UCL intensities compared to a reference cell culture incubated with UCNPs using a 1000 times lower concentration of MIMs.

\subsection{Dielectric nanostructures}

Surface plasmons are highly effective in localizing light and creating a strong local electric field, which can be used to enhance upconversion. However, the mode volume where electric field is enhanced tends to be small and thus enhancement over large areas is generally difficult to achieve. Also, metals inevitably quench luminescence by 
A

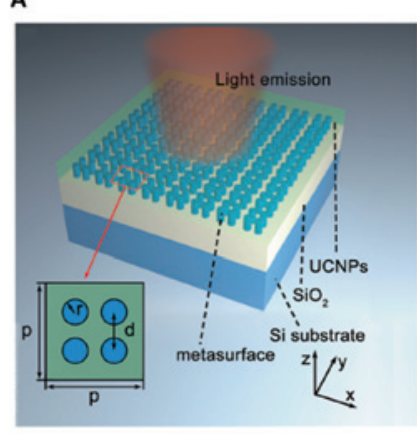

B

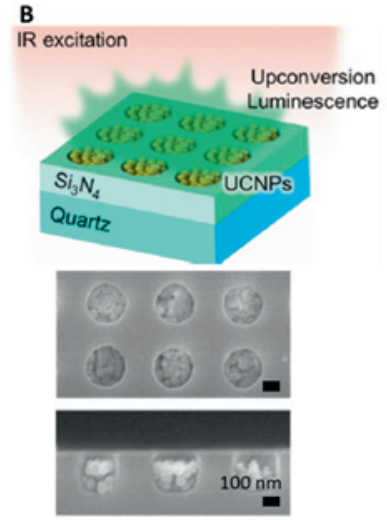

C

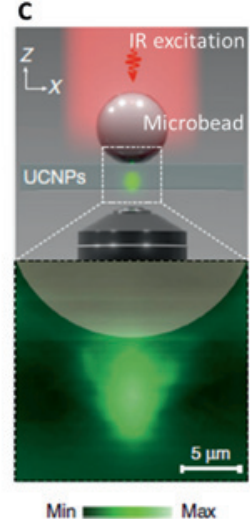

Figure 5: Dielectric nanostructures with UCNPs. (A) Schematic of the dual resonance all-dielectric metasurface. Each unit cell of the metasurface is a square consisting of four silicon cylinders. These cylinders have the same radius and the distance in each unit cell. UCNPs are dispersed uniformly on the metasurface. A magnified top view of a unit cell is shown in the inset. (B) Top: Schematic of dielectric photonic crystal structure with $\mathrm{SiO}_{2}$ coated $\mathrm{NaYF}_{4}: \mathrm{Yb}^{3+}, \mathrm{Er}^{3+}$ UCNPs. The middle and bottom images are the top-down and cross-sectional SEM images of UCNP-filled photonic crystal. (C) Illustration of a microbead lensing effect in

the $\mathrm{NaYF}_{4}: \mathrm{Yb}^{3+} \mathrm{Er}^{3+}$-embedded polydimethylsiloxane (PDMS) film. The UCNP-embedded PDMS substrate is placed underneath a $20 \mu \mathrm{m}$ dielectric microbead. Reproduced from Ref. [72] for (A) with permission from The Royal Society of Chemistry, Ref. [73] for (B) with permission from Mao et al., ACS Photon. vol. 6, pp. 1882-1888, 2019, ( 2019 by the American Chemical Society, and Ref. [74] for (C) with permission from Liang et al., Nat. Commun. vol. 10, p. 1391, 2019, licensed under Creative Commons Attribution 4.0 International License.

providing additional non-radiative recombination pathways. When the UCNPs are close to the metallic surface, the quantum efficiency drops rapidly due to this quenching effect. The development of high permittivity dielectric nanostructures gives an alternative approach to enhance the fluorescence signals [72-76]. Electric and magnetic resonances in dielectric nanostructures create strong localized optical fields which, although generally weaker than in plasmonic nanostructures, benefit from less quenching.

Dielectric photonic crystal (PhC) structures with UCNPs provide an excellent alternative to plasmonic designs. By using band edge states, it is possible to excite optical resonances extended over the entire photonic structure, thereby achieving field enhancement over a large area [75, 76]. Moreover, PhC structures are suitable for applications like lighting, displays, and sensors that may require a large active area. Transparent dielectric microbead lens have also been used to focus an incident light beam into a subwavelength photonic hotspot, enabling efficient photon upconversion under low illumination.

Gong et al. introduced a dual-resonance all-dielectric metasurface with periodic silicon pillar arrays to enhance the signals emitted by $\mathrm{NaYF}_{4}: \mathrm{Yb}^{3+}, \mathrm{Tm}^{3+}$ nanoparticles (Figure 5A) [72]. The electric and magnetic dipole resonances of the metasurface were designed to enhance the local field near the silicon pillars. The magnetic and electric dipole resonances were tuned to match the luminescence peak at $800 \mathrm{~nm}$ and the wavelength of the excitation laser $(965 \mathrm{~nm})$, respectively. The two resonances can be tuned separately because the resonance wavelength of the magnetic dipole mode was mainly dependent on radius of pillars, while the electric dipole mode was dependent on both the radius and thickness of the cylinders.
Furthermore, the collection efficiency was enhanced due to the directional emission of the nanostructure. A total upconversion signal enhancement of 400x was achieved.

Our group incorporated UCNPs in a two-dimensional planar $\mathrm{PhC}$ structure for upconversion enhancement (Figure 5B) [73]. UNCPs were filled inside the nanoholes of the $\mathrm{Si}_{3} \mathrm{~N}_{4} \mathrm{PhC}$ structure with a self-assembly method. The local field enhancement experienced by the UCNPs in the nanoholes resulted in overall enhancement factors of 130x for green and 350x for red luminescence. The local field enhancement in the dielectric PhC structure was not as high as plasmonic nanostructures but this lower field enhancement was compensated by the fact that the purely dielectric PhC structure causes much less quenching than the plasmonic structures made of metal and was achieved over a large area.

Finally, Liang et al. used a dielectric microbead lens to achieve large UCL enhancement as shown in Figure 5C [74]. In this scheme, the intensity of the UCL could be enhanced by focusing both excitation and emission fields using dielectric microbeads. $\mathrm{NaYF}_{4}: \mathrm{Yb}^{3+}, \mathrm{Er}^{3+} @ \mathrm{NaYF}_{4}$ and NaGd$\mathrm{F}_{4}: \mathrm{Yb}^{3+} / \mathrm{Tm}^{3+} @ \mathrm{NaGdF}_{4}: \mathrm{Eu}^{3+}$ were investigated and the enhancement factor was 103,000x for $\mathrm{Eu}^{3+}$ at $614 \mathrm{~nm}$ and 100x for $\mathrm{Er}^{3+}$ at $540 \mathrm{~nm}$, respectively. The extremely high enhancement of $\mathrm{Eu}^{3+}$ at $614 \mathrm{~nm}$ was due to the involvement of four photons for the upconversion. As discussed in section 2, the overall upconversion is improved much more in the higher order upconversion system. The authors additionally provided experimental results for enabling two- or multiphoton pumped upconversion for document security and optogenetic applications. For document security, an encrypted code was printed on a piece of paper using $\mathrm{NaYF}_{4}: \mathrm{Yb}^{3+}, \mathrm{Er}^{3+} @ \mathrm{NaYF}_{4}$ nanoparticle inks and a 
PDMS sheet and then decoded with a tungsten lamp at subsolar irradiance. For optogenetic application, a flexible PDMS chip with microbeads and $\mathrm{NaYF}_{4}: \mathrm{Yb}^{3+}, \mathrm{Tm}^{3+} @ \mathrm{NaYF}_{4}$ nanoparticles were placed underneath a brain section and then the electrophysiological response of neurons containing melanin-concentrating hormone was recorded.

\section{Conclusions and outlook}

The field of luminescence upconversion has experienced a remarkable growth in the past decade. Significant progress has been made in the development of refined synthesis methods that yield high quality UCNPs. Aided by the theoretical studies on the energy transfer and quenching mechanisms, novel designs such as core-shell have been used to improve upconversion efficiency [13-22]. Complementary to the chemistry-based approaches for improving upconversion, nanophotonics offer pathways to a significant boost in upconversion luminescence [45-76]. Engineering photonic states, which can be done without affecting the electronic states, can make a dramatic impact on the light-matter interaction in general. In the context of upconversion enhancement, nanophotonic approaches can be used to enhance all processes involved in upconversion: absorption, energy transfer and emission. Quantum electrodynamics provides a firm theoretical basis to manipulate these processes while the interactions among the various energy levels are described well by the rate equation analysis. These strong theoretical foundations allow rational design of nanostructures tailored to enhance specific processes as desired.

From the fabrication standpoint, the wealth of nanofabrication techniques allows precise fabrication of complex nanostructures. Nanolithography techniques such as electron-beam lithography are highly effective in producing nanoscale features. However, they are generally limited to small areas and planar geometries and thus may not be suitable for applications requiring low-cost fabrication over large areas, such as biosensing. On the other hand, bottom-up approaches such as colloidal self-assembly can produce large area and often three-dimensional structures. These techniques however tend to produce a larger degree of disorder and it is generally difficult to achieve precise control over the nanoscale geometry to which nanophotonic enhancement is sensitive. It is therefore desirable to develop a hybrid approach that exploits the strengths of multiple fabrication techniques. A prime example is the combination of laser interference lithography and self-assembly that has been used to fabricate MIM structures and achieved 3 orders of magnitude enhancement. Further development of novel fabrication techniques will be critical to the continued progress in nanophotonically enhanced upconversion materials.

Through a combination of a robust understanding of the theoretical underpinnings and the sophisticated design and fabrication of nanostructures, it is possible to greatly enhance the upconversion process. In the last few years in particular, we see reported enhancements that, for the first time, exceed three orders of magnitude. This is particularly impressive for dispersible nanostructures for biological applications where the design constraints make this especially challenging. We observe a noticeable shift to absorption enhancement that utilizes the nonlinear scaling achievable by enhancing the absorption cross-section of the nanostructures. In addition to the traditional gold and silver nanostructures, new exotic materials and more complicated designs are starting to be utilized to enhance upconversion. We anticipate new strides will be made in the use of dielectric nanostructures where the lack of plasmonic quenching may allow for greater enhancement. We also predict further work into the enhancement of multiphoton upconversion processes such as those involved in $\mathrm{Tm}^{3+}$ or $\mathrm{Eu}^{3+}$. While these are less efficient than the traditional erbium-based two photon process, the higher order power dependence of these processes should result in a much larger enhancement in upconversion emission.

From the application standpoint, we anticipate continued focus on biological and medical applications. UCNP's intrinsic advantages such as no background autofluorescence, minimal background tissue damage and long penetration depth in biological tissues will keep UCNPs on the forefront of biological imaging and therapeutics. UCNPs are particularly attractive for in vivo applications where the safe level of laser power is quite small [12,13] and thus other nonlinear probes such as two-photon dyes are not usable. For wide-spread use in in vivo settings, the upconversion efficiency must be further improved. It is also important to develop surface treatment techniques for targeted delivery. With the current pace of developments, it is conceivable UCNPs will soon be placed among the mainstream nanoprobes commonly used in both laboratories and clinics.

\section{References}

[1] F. Auzel, "Upconversion and anti-stokes processes with $\mathrm{f}$ and d ions in solids," Chem. Rev., vol. 104, pp. 139-174, 2004.

[2] M. Haase, H. Schäfer, “Upconverting nanoparticles," Angew. Chemie Int. Ed., vol. 50, pp. 5808-5829, 2011.

[3] R. Deng, F. Qin, R. Chen, W. Huang, M. Hong, X. Liu, "Temporal full-colour tuning through non-steady-state upconversion," Nat. Nanotechnol., vol. 10, pp. 237-242, 2015. 
[4] X. Liu, Y. Wang, X. Li, et al., "Binary temporal upconversion codes of $\mathrm{Mn}^{2+}$-activated nanoparticles for multilevel anticounterfeiting," Nat. Commun., vol. 8, pp. 899, 2017.

[5] M. Wang, M. Li, A. Yu, J. Wu, C. Mao, "Rare earth fluorescent nanomaterials for enhanced development of latent fingerprints," ACS Appl. Mater. Interfaces, vol. 7, pp. 2811028115, 2015.

[6] X. Wang, Q. Liu, Y. Bu, C. S. Liu, T. Liu, X. Yan, "Optical temperature sensing of rare-earth ion doped phosphors," RSC Adv., vol. 5, pp. 86219-86236, 2015.

[7] M. He, X. Pang, X. Liu, et al., "Monodisperse dual-functional upconversion nanoparticles enabled near-infrared organolead halide perovskite solar cells," Angew. Chemie Int. Ed., vol. 55, pp. 4280-4284, 2016.

[8] J. Reszczyńska, T. Grzyb, J. W. Sobczak, et al., "Visible light activity of rare earth metal doped $\left(\mathrm{Er}^{3+}, \mathrm{Yb}^{3+}\right.$ or $\left.\mathrm{Er}^{3+} / \mathrm{Yb}^{3+}\right)$ titania photocatalysts," Appl. Catal. B Environ., vol. 163, pp. 40-49, 2015.

[9] L. Feng, F. He, B. Liu, et al., " $\mathrm{g}-\mathrm{C}_{3} \mathrm{~N}_{4}$ coated upconversion nanoparticles for $808 \mathrm{~nm}$ near-infrared light triggered phototherapy and multiple imaging," Chem. Mater., vol. 28, pp. 7935-7946, 2016.

[10] L. He, J. Dragavon, S. Cho, et al., "Self-assembled gold nanostar$\mathrm{NaYF}^{4}: \mathrm{Yb} /$ Er clusters for multimodal imaging, photothermal and photodynamic therapy," J. Mater. Chem. B, vol. 4, pp. 44554461, 2016.

[11] K. Li, Q. Su, W. Yuan, et al., "Ratiometric monitoring of intracellular drug release by an upconversion drug delivery nanosystem," ACS Appl. Mater. Interfaces, vol. 7, pp. 1227812286, 2015.

[12] S. A. Hilderbrand, F. Shao, C. Salthouse, U. Mahmood, R. Weissleder, "Upconverting luminescent nanomaterials: application to in vivo bioimaging," Chem. Commun., vol. 28, pp. 4188-4190, 2009.

[13] N. Möller, T. Hellwig, L. Stricker, S. Engel, C. Fallnich, BJ. Ravoo, "Near-infrared photoswitching of cyclodextrin-guest complexes using lanthanide-doped $\mathrm{LiYF}_{4}$ upconversion nanoparticles," Chem. Commun., vol. 53, pp. 240-243, 2017.

[14] A. R. Hong, S. Y. Kim, S. H. Cho, K. Lee, H. S. Jang, "Facile synthesis of multicolor tunable ultrasmall LiYF $4: \mathrm{Yb}, \mathrm{Tm}, \mathrm{Er} / \mathrm{LiGdF}_{4}$ core/shell upconversion nanophosphors with sub-10 nm size," Dye Pigment, vol. 139, pp. 831-838, 2017.

[15] X. Du, X. Wang, L. Meng, Y. Bu, X. Yan, “Enhance the $\mathrm{Er}^{3+}$ upconversion luminescence by constructing $\mathrm{NaGdF}_{4}: \mathrm{Er}^{3+} @ \mathrm{NaGdF}_{4}$ : $\mathrm{Er}^{3+}$ active-core/active-shell nanocrystals," Nanoscale Res. Lett., vol. 12, p. 163, 2017.

[16] J. Liu, G. Chen, S. Hao, C. Yang, "Sub-6 nm monodisperse hexagonal core/shell $\mathrm{NaGdF}_{4}$ nanocrystals with enhanced upconversion photoluminescence," Nanoscale, vol. 9, pp. 9198, 2017.

[17] D. Li, X. Liu, J. Qiu, “Probing interaction distance of surface quenchers in lanthanide-doped upconversion core-shell nanoparticles,”J. Phys. Chem. C, vol. 122, pp. 10278-10283, 2018.

[18] B. Shen, S. Cheng, Y. Gu, et al., "Revisiting the optimized doping ratio in core/shell nanostructured upconversion particles," Nanoscale, vol. 9, pp. 1964-1971, 2017.

[19] NJJ. Johnson, S. He, S. Diao, EM. Chan, H. Dai, A. Almutairi, "Direct evidence for coupled surface and concentration quenching dynamics in lanthanide-doped nanocrystals," J. Am. Chem. Soc., vol. 139, pp. 3275-3282, 2017.
[20] X. Huang, "Giant enhancement of upconversion emission in $\left(\mathrm{NaYF}_{4}: \mathrm{Nd}^{3+} / \mathrm{Yb}^{3+} / \mathrm{Ho}^{3+}\right) /\left(\mathrm{NaYF}_{4}: \mathrm{Nd}^{3}+/ \mathrm{Yb}^{3}+\right)$ core/shell nanoparticles excited at $808 \mathrm{~nm}$," Opt. Lett., vol. 40, pp. 3599-3602, 2015.

[21] X. Huang, J. Lin, “Active-core/active-shell nanostructured design: an effective strategy to enhance $\mathrm{Nd}^{3+} / \mathrm{Yb}^{3+}$ cascade sensitized upconversion luminescence in lanthanide-doped nanoparticles," J. Mater. Chem. C, vol. 3, pp. 7652-7657, 2015.

[22] K. Huang, H. Liu, M. Kraft, et al., "A protected excitation-energy reservoir for efficient upconversion luminescence," Nanoscale, vol. 10, pp. 250-259, 2018.

[23] S. Hao, G. Chen, C. Yang, et al., " $\mathrm{Nd}^{3+}$-Sensitized multicolor upconversion luminescence from a sandwiched core/shell/shell nanostructure," Nanoscale, vol. 9, pp. 10633-10638, 2017.

[24] W. Shao, G. Chen, A. Kuzmin, et al., "Tunable narrow band emissions from dye-sensitized core/shell/shell nanocrystals in the second near-infrared biological window," J. Am. Chem. Soc., vol. 138, pp. 16192-16195, 2016.

[25] W. Zou, C. Visser, J. A. Maduro, M. S. Pshenichnikov, J. C. Hummelen, "Broadband dye-sensitized upconversion of nearinfrared light," Nat. Photonics, vol. 6, pp. 560-564, 2012.

[26] G. Chen, J. Damasco, H. Qiu, et al., "Energy-cascaded upconversion in an organic dye-sensitized core/shell fluoride nanocrystal,” Nano. Lett., vol. 15, pp. 7400-7407, 2015.

[27] P. Lei, P. Zhang, S. Yao, et al., "Optimization of $\mathrm{Bi}^{3+}$ in upconversion nanoparticles induced simultaneous enhancement of near-infrared optical and x-ray computed tomography imaging capability," ACS Appl. Mater. Interfaces, vol. 8, pp. 27490-27497, 2016.

[28] Y.Zhu, S. Zhao, B. Zhou, H. Zhu, Y. Wang, "Enhancing upconversion luminescence of $\mathrm{LiYF}_{4}: \mathrm{Yb}$,Er nanocrystals by $\mathrm{Cd}^{2+}$ doping and coreshell structure,”J. Phys. Chem. C, vol. 121, pp. 18909-18916, 2017.

[29] J. Tang, L. Chen, J. Li, et al., "Selectively enhanced red upconversion luminescence and phase/size manipulation via $\mathrm{Fe}^{3+}$ doping in $\mathrm{NaYF}_{4}: \mathrm{Yb}$,Er nanocrystals," Nanoscale, vol. 7, pp. 14752-14759, 2015.

[30] X. Chuai, X. Guo, X. Liu, et al., "Bifunctional NaGdF 4 :Yb, Er, Fe nanocrystals with the enhanced upconversion fluorescence," Opt. Mater. (Amst), vol. 44, pp. 13-17, 2015.

[31] M. Ding, D. Chen, S. Yin, et al., "Simultaneous morphology manipulation and upconversion luminescence enhancement of $\beta-\mathrm{NaYF}_{4}: \mathrm{Yb}^{3+} / \mathrm{Er}^{3+}$ microcrystals by simply tuning the KF dosage," Sci. Rep., vol. 5, Art no. 12745, 2015.

[32] L. Mukhopadhyay, V. K. Rai, R. Bokolia, K Sreenivas, “980nm excited $\mathrm{Er}^{3+} / \mathrm{Yb}^{3+} / \mathrm{Li}^{+} / \mathrm{Ba}^{2+}: \mathrm{NaZnPO}_{4}$ upconverting phosphors in optical thermometry," J. Lumin., vol. 187, pp. 368-377, 2017.

[33] L. Mukhopadhyay, V. Kumar Rai, "Upconversion based near white light emission, intrinsic optical bistability and temperature sensing in $\mathrm{Er}^{3+} / \mathrm{Tm}^{3+} / \mathrm{Yb}^{3+} / \mathrm{Li}^{+}: \mathrm{NaZnPO}_{4}$ phosphors," New. J. Chem., vol. 41, pp. 7650-7661, 2017.

[34] M. Ding, Y. Ni, Y. Song, et al., " $\mathrm{Li}^{+}$ions doping core-shell nanostructures: An approach to significantly enhance upconversion luminescence of lanthanide-doped nanocrystals," J. Alloys Compd., vol. 623, pp. 42-48, 2015.

[35] W. Park, D. Lu, S. Ahn, "Plasmon enhancement of luminescence upconversion," Chem. Soc. Rev., vol. 44, pp. 2940-2962, 2015. 
[36] E. M. Purcell, H. C. Torrey, R. V. Pound, "Resonance absorption by nuclear magnetic moments in a solid," Phys. Rev, vol. 69, pp. 37-38, 1946.

[37] S. Haroche, D. Kleppner, "Cavity quantum electrodynamics," Phys. Today, vol. 42, pp. 24-30, 1989.

[38] P. Anger, P. Bharadwaj, L. Novotny, "Enhancement and quenching of single-molecule fluorescence," Phys. Rev. Lett., vol. 96, Art no. 113002, 2006.

[39] D. Lu, S. K. Cho, S. Ahn, L. Brun, C. J. Summers, W. Park, "Plasmon enhancement mechanism for the upconversion processes in $\mathrm{NaYF}_{4}: \mathrm{Yb}^{3+}, \mathrm{Er}^{3+}$ nanoparticles: Maxwell versus Förster," ACS Nano., vol. 8, pp. 7780-7792, 2014.

[40] J. R. Lakowicz, "Radiative decay engineering 5: metalenhanced fluorescence and plasmon emission," Anal. Biochem., vol. 337, pp. 171-194, 2005.

[41] P. Andrew, W. L. Barnes, "Förster energy transfer in an optical microcavity,” Science, vol. 290, pp. 785-788, 2000.

[42] H. T. Dung, L. Knöll, D. G. Welsch, "Intermolecular energy transfer in the presence of dispersing and absorbing media," Phys. Rev. A, vol. 65, Art no. 43813, 2002.

[43] M. J. A. de Dood, J. Knoester, A. Tip, A. Polman, "Förster transfer and the local optical density of states in erbium-doped silica," Phys. Rev. B, vol. 71, Art no. 115102, 2005.

[44] C. Blum, N. Zijlstra, A. Lagendijk, et al., "Nanophotonic control of the Förster resonance energy transfer efficiency," Phys. Rev. Lett., vol. 109, Art no. 203601, 2012.

[45] D. Lu, C. Mao, SK. Cho, S. Ahn, W. Park, "Experimental demonstration of plasmon enhanced energy transfer rate in $\mathrm{NaYF}_{4}: \mathrm{Yb}^{3+}, \mathrm{Er}^{3+}$ upconversion nanoparticles," Sci. Rep., vol. 6, Art no. 18894, 2016.

[46] X. Chen, W. Xu, L. Zhang, et al., "Large upconversion enhancement in the 'islands' Au-Ag alloy/ $\mathrm{NaYF}_{4}: \mathrm{Yb}^{3+}, \mathrm{Tm}^{3+} / \mathrm{Er}^{3+}$ composite films, and fingerprint identification," Adv. Funct. Mater., vol. 25, pp. 5462-5471, 2015.

[47] KT. Lee, JH. Park, SJ. Kwon, et al., "Simultaneous enhancement of upconversion and downshifting luminescence via plasmonic structure," Nano. Lett., vol. 15, pp. 2491-2497, 2015.

[48] Z. Zhang, Y. Liu, Y. Fang, et al., "Near-infrared-plasmonic energy upconversion in a nonmetallic heterostructure for efficient $\mathrm{H}_{2}$ evolution from ammonia borane," Adv. Sci., vol. 5, Art no. 1800748, 2018.

[49] Y. Zhu, W. Xu, G. Li, S. Cui, X. Liu, H. Song, “Plasmonic enhancement of the upconversion fluorescence in $\mathrm{YVO}_{4}: \mathrm{Yb}^{3+}, \mathrm{Er}^{3+}$ nanocrystals based on the porous Ag film," Nanotechnology, vol. 26, Art no. 145602, 2015.

[50] D. Zhou, D. Liu, J. Jin, et al., "Semiconductor plasmon-sensitized broadband upconversion and its enhancement effect on the power conversion efficiency of perovskite solar cells," J. Mater. Chem. A, vol. 5, pp. 16559-16567, 2017.

[51] D. Zhou, D. Li, X. Zhou, et al., "Semiconductor plasmon induced up-conversion enhancement in $\mathrm{mCu}_{2-\mathrm{x}} \mathrm{S} @ \mathrm{SiO} @ \mathrm{Y}_{2} \mathrm{O}_{3}: \mathrm{Yb}^{3+} / \mathrm{Er}^{3+}$ core-shell nanocomposites," ACS Appl. Mater. Interfaces, vol. 9, pp. 35226-35233, 2017.

[52] D. Zhou, D. Liu, W. Xu, et al., “Observation of considerable upconversion enhancement induced by $\mathrm{Cu}_{2-\mathrm{x}} \mathrm{S}$ plasmon nanoparticles," ACS Nano., vol. 10, pp. 5169-5179, 2016.

[53] W. Zhang, J. Li, H. Lei, B. Li, "Plasmon-induced selective enhancement of green emission in lanthanide-doped manoparticles," ACS Appl. Mater. Interfaces, vol. 9, pp. 4293542942, 2017.
[54] Z. Yin, H Li, W. Xu, et al., "Local field modulation induced threeorder upconversion enhancement: combining surface plasmon effect and photonic crystal effect," Adv. Mater., vol. 28, pp. 2518-2525, 2016.

[55] A. Ling Feng, M. Lin, L. Tian, et al., "Selective enhancement of red emission from upconversion nanoparticles via surface plasmoncoupled emission," RSC Adv., vol. 5, pp. 76825-76835, 2015.

[56] Z. Yin, D. Zhou, W. Xu, et al., "Plasmon-enhanced upconversion luminescence on vertically aligned gold nanorod monolayer supercrystals," ACS Appl. Mater. Interfaces, vol. 8, pp. 1166711674, 2016.

[57] A. L. Feng, M. L. You, L. Tian, et al., "Distance-dependent plasmon-enhanced fluorescence of upconversion nanoparticles using polyelectrolyte multilayers as tunable spacers," Sci. Rep., vol. 5, p. 7779, 2015.

[58] R. Viktoria Manurung, C. Ting Wu, P. Kumar Roy, S. Chattopadhyay, “A plasmon-tuned 'gold sandwich' for metal enhanced fluorescence in silica coated $\mathrm{NaYF}_{4}: \mathrm{Yb}, \mathrm{Er}$ upconversion nanoparticles," RSC Adv., vol. 6, pp. 8708887095, 2016.

[59] J. Fisher, B. Zhao, C. Lin, M. Berry, P. S. May, S. Smith, "Spectroscopic imaging and power dependence of near-infrared to visible upconversion luminescence from $\mathrm{NaYF}_{4}: \mathrm{Yb}^{3+}, \mathrm{Er}^{3+}$ nanoparticles on nanocavity arrays," J. Phys. Chem. C, vol. 119, pp. 24976-24982, 2015.

[60] Y. L. Wang, N Mohammadi Estakhri, A. Johnson, et al., "Tailoring plasmonic enhanced upconversion in single $\mathrm{NaYF}_{4}: \mathrm{Yb}^{3+} / \mathrm{Er}^{3+}$ nanocrystals," Sci. Rep., vol. 5, Art no. 10196, 2015.

[61] S. Rohani, M. Quintanilla, S. Tuccio, et al., "Enhanced luminescence, collective heating, and nanothermometry in an ensemble system composed of lanthanide-doped upconverting nanoparticles and gold nanorods," Adv. Opt. Mater., vol. 3, pp. 1606-1613, 2015.

[62] B. Shao, Z. Yang, J. Li, et al., "Upconversion emission enhancement by porous silver films with ultra-broad plasmon absorption," Opt. Mater. Express, vol. 7, pp. 1188-1197, 2017.

[63] B. Shao, Z. Yang, Y. Wang, et al., "Coupling of Ag nanoparticle with inverse opal photonic crystals as a novel strategy for upconversion emission enhancement of $\mathrm{NaYF}_{4}: \mathrm{Yb}^{3+}, \mathrm{Er}^{3+}$ nanoparticles," ACS Appl. Mater. Interfaces, vol. 7, pp. 25211-25218, 2015.

[64] K. Yamamoto, M. Fujii, S. Sowa, K. Imakita, K. Aoki, "Upconversion luminescence of rare-earth-doped $\mathrm{Y}_{2} \mathrm{O}_{3}$ nanoparticle with metal nano-cap,” J. Phys. Chem. C, vol. 119, pp. 1175-1179, 2015.

[65] X. Chen, D. Zhou, W. Xu, et al., "Fabrication of Au-Ag nanocage@NaYF $\mathrm{NNaYF}_{4}: \mathrm{Yb}, \mathrm{Er}$ core-shell hybrid and its tunable upconversion enhancement," Sci. Rep., vol. 7, Art no. 41079, 2017.

[66] A. Das, C. Mao, S. Cho, K. Kim, W. Park, "Over 1000-fold enhancement of upconversion luminescence using waterdispersible metal-insulator-metal nanostructures," Nat. Commun., vol. 9, p. 4828, 2018.

[67] Y. Qin, Z. Dong, D. Zhou, Y. Yang, X. Xu, J. Qiu, “Modification on populating paths of $\beta-\mathrm{NaYF}_{4}: \mathrm{Nd} / \mathrm{Yb} / \mathrm{Ho} @ \mathrm{SiO}_{2} @ A g$ core/doubleshell nanocomposites with plasmon enhanced upconversion emission," Opt. Mater. Express, vol. 6, pp. 1942-1955, 2016.

[68] S. K. Maurya, S. P. Tiwari, A. Kumar, K. Kumar, "Plasmonic

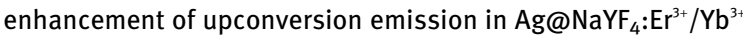
phosphor," J. Rare Earths, vol. 36, pp. 903-910, 2018.

[69] E. J. He, M. Moskovits, J. Dong, et al., "Luminescence enhancement mechanism of lanthanide-doped hybrid 
nanostructures decorated by silver nanocrystals," Plasmonics, vol. 10, pp. 357-368, 2015.

[70] K. Green, J. Wirth, S. F. Lim, “Optical investigation of gold shell enhanced $25 \mathrm{~nm}$ diameter upconverted fluorescence emission," Nanotechnology, vol. 27, Art no. 135201, 2016.

[71] J. He, W. Zheng, F. Ligmajer, et al., "Plasmonic enhancement and polarization dependence of nonlinear upconversion emissions from single gold nanorod@ $\mathrm{SiO}_{2} @ \mathrm{CaF}_{2}: \mathrm{Yb}^{3+}, \mathrm{Er}^{3+}$ hybrid coreshell-satellite nanostructures," Light Sci. Appl., vol. 6, e16217e16217, 2017.

[72] C. Gong, W. Liu, N. He, H. Dong, Y. Jin, S. He, “Upconversion enhancement by a dual-resonance all-dielectric metasurface," Nanoscale, vol. 11, pp. 1856-1862, 2019.
[73] C. Mao, K. Min, K. Bae, et al., "Enhanced upconversion luminescence by two-dimensional photonic crystal structure," ACS Photonics, vol. 6, pp. 1882-1888, 2019.

[74] L. Liang, D. B. L. Teh, N. D. Dinh, et al., “Upconversion amplification through dielectric superlensing modulation," Nat. Commun., vol. 10, pp. 1391, 2019.

[75] K. Min, H. Jung, Y. Park, et al., "A colloidal quantum dot photonic crystal phosphor: nanostructural engineering of the phosphor for enhanced color conversion," Nanoscale, vol. 9, pp. 87038709, 2017.

[76] J. Lee, K. Min, Y. Park, K. S. Cho, H. Jeon, "Photonic crystal phosphors integrated on a blue LED chip for efficient white light generation," Adv. Mater., vol. 30, Art no. 1703506, 2018. 